\title{
Efficient Type-II Heterojunction Nanorod Sensitized Solar Cells Realized by Controlled Synthesis of Core/Patchy-Shell Structure and CdS Cosensitization
}

\author{
Sangheon Lee, ${ }^{a+}{ }^{+}$Joseph C. Flanagan, ${ }^{b,+}$ Jaewook Kim, ${ }^{a}$ Alan Jiwan Yun, ${ }^{a}$ Byungho Lee, ${ }^{a}$ \\ Moonsub Shim, ${ }^{b, *}$ and Byungwoo Park ${ }^{a, *}$ \\ a WCU Hybrid Materials Program, \\ Department of Materials Science and Engineering, Research Institute of Advanced Materials, \\ Seoul National University, Seoul 08226, Korea. \\ ${ }^{b}$ Department of Materials Science and Engineering and Frederick Seitz Materials Research Laboratory, \\ University of Illinois at Urbana-Champaign, Urbana, Illinois 61801, United States.
}
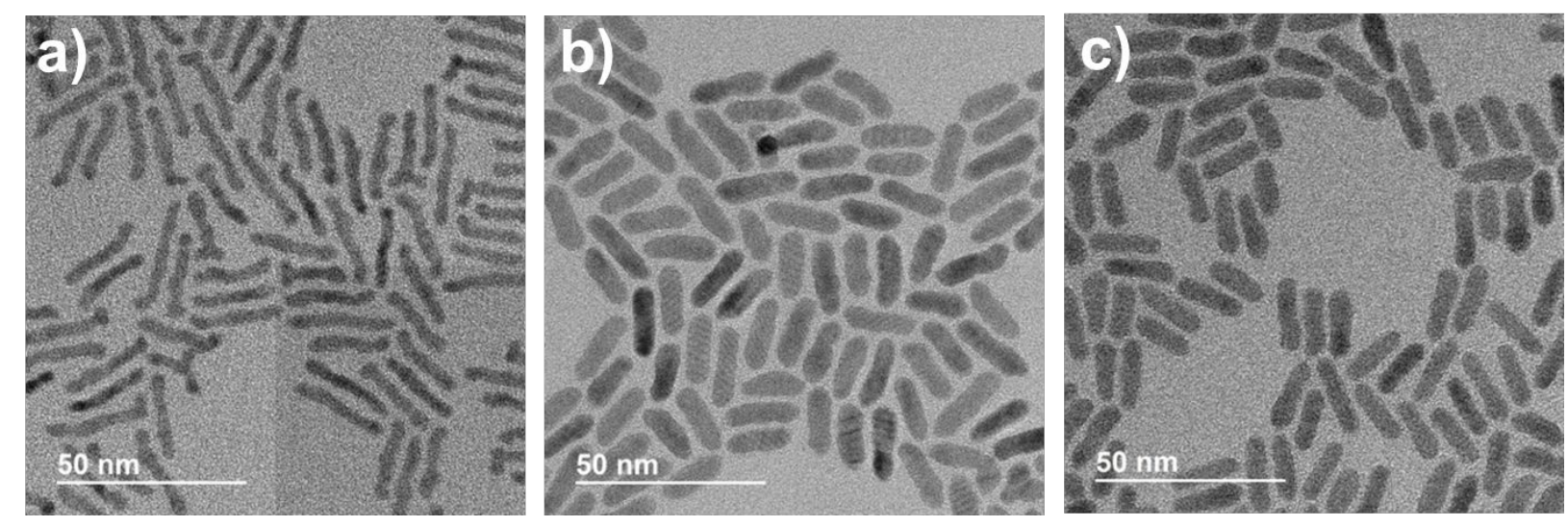

Figure S1. Representative low-magnification TEM images of (a) linear heterojunction nanorods (lHNRs), (b) core-shell heterojunction nanorods ( $c$ HNRs), and (c) core/patchy-shell heterojunction nanorods ( $p \mathrm{HNRs})$.

\footnotetext{
* Corresponding author. E-mail: mshim@illinois.edu; Phone: +1-217-333-7361.

* Corresponding author. E-mail: byungwoo@snu.ac.kr; Phone: +82-2-880-8319.

+ These two authors contributed equally to this work.
} 

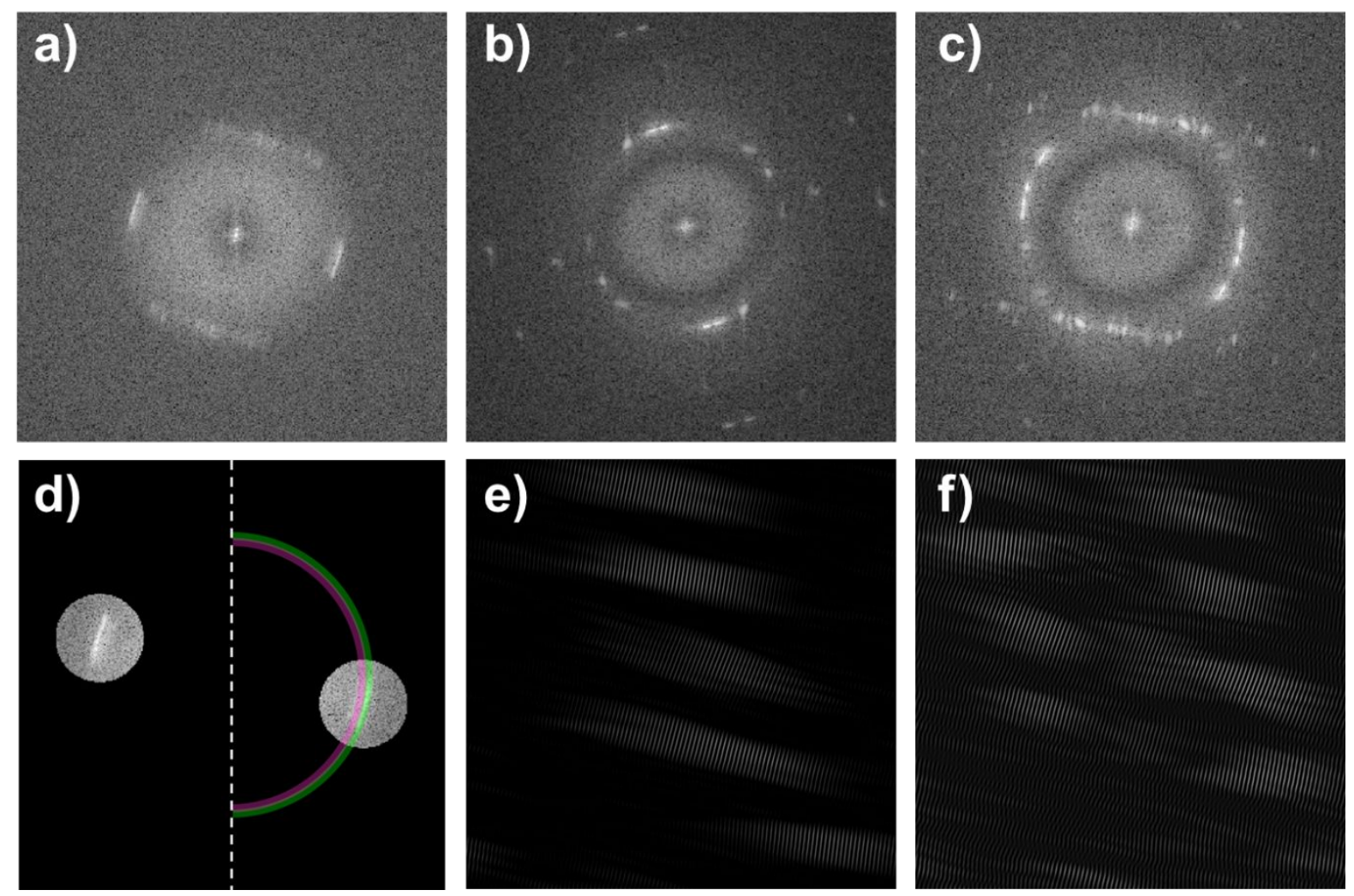

Figure S2. FFTs for each of the TEM images in main Fig. 1(b) are shown here in (a-c) ( $l \mathrm{HNR}, c \mathrm{HNR}$, and $p \mathrm{HNR}$ respectively). The light bands in the FFT indicate periodicity in the source image. In this case, the periodicity comes from the lattice fringes along the nanorods. The further from the center, the smaller the period, so in principle, CdSe and $\mathrm{CdSe}_{x} \mathrm{Te}_{1-x}$ can be separated based on the difference in lattice parameter. An example is shown in (d) for $l$ HNR. First, the region of interest corresponding to the [0001] lattice fringes is isolated from the full FFT (left). Then, two circular masks of different diameter are applied (right). The inverse FFT (IFFT) can then be taken to reconstruct the real-space image. The location of CdSe corresponds to the IFFT of the outer masked area, shown in (e). The location of $\mathrm{CdSe}_{x} \mathrm{Te}_{1-x}$ corresponds to the IFFT of the inner masked area, shown in (f). The individual components can then be assigned color and overlaid to finish the reconstruction. Pure green and magenta in the final image (Fig. 1(b)) are relatively unstrained CdSe and $\mathrm{CdSe}_{x} \mathrm{Te}_{1-x}$, and white regions are where they overlap, indicating intermediate lattice parameter. The analysis is relatively straightforward in the case of $l \mathrm{HNR}$, as the two components have a small interface and separate regions can be identified on the FFT. For $c \mathrm{HNR}$ and $p \mathrm{HNR}$ though, the interfacial area is large and strain distorts the lattice parameter. The result in the FFT is one wider band. Therefore, the masks were applied to split this band down the center, identifying regions with smaller-than-average and larger-than-average lattice

parameter. 
(a)

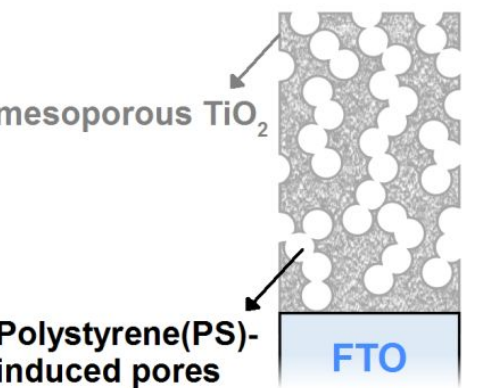

$0 / 3$

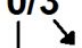

\# of tapes used to coat the PS-incorporated $\mathrm{TiO}_{2}$ paste

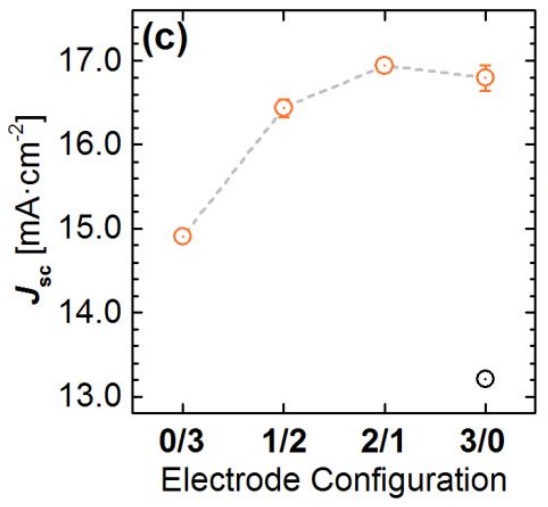

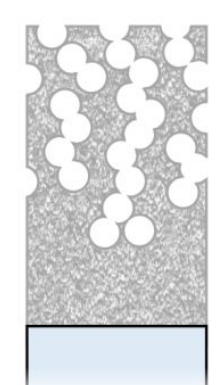

$1 / 2$

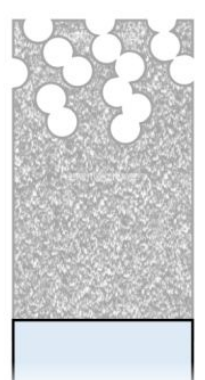

2/1

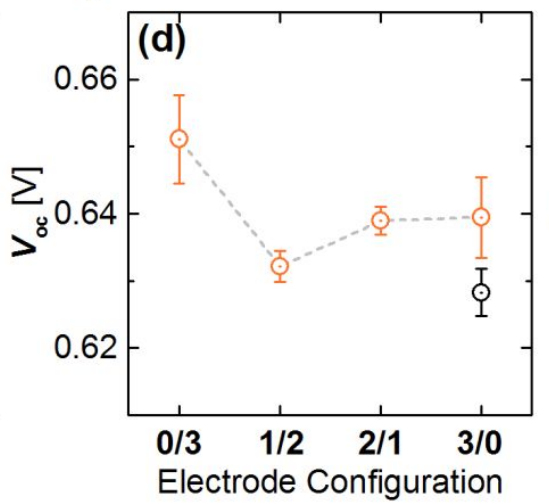

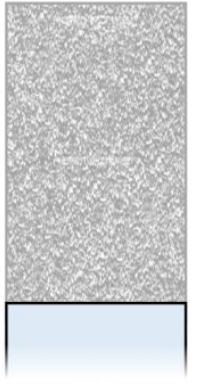

$3 / 0$
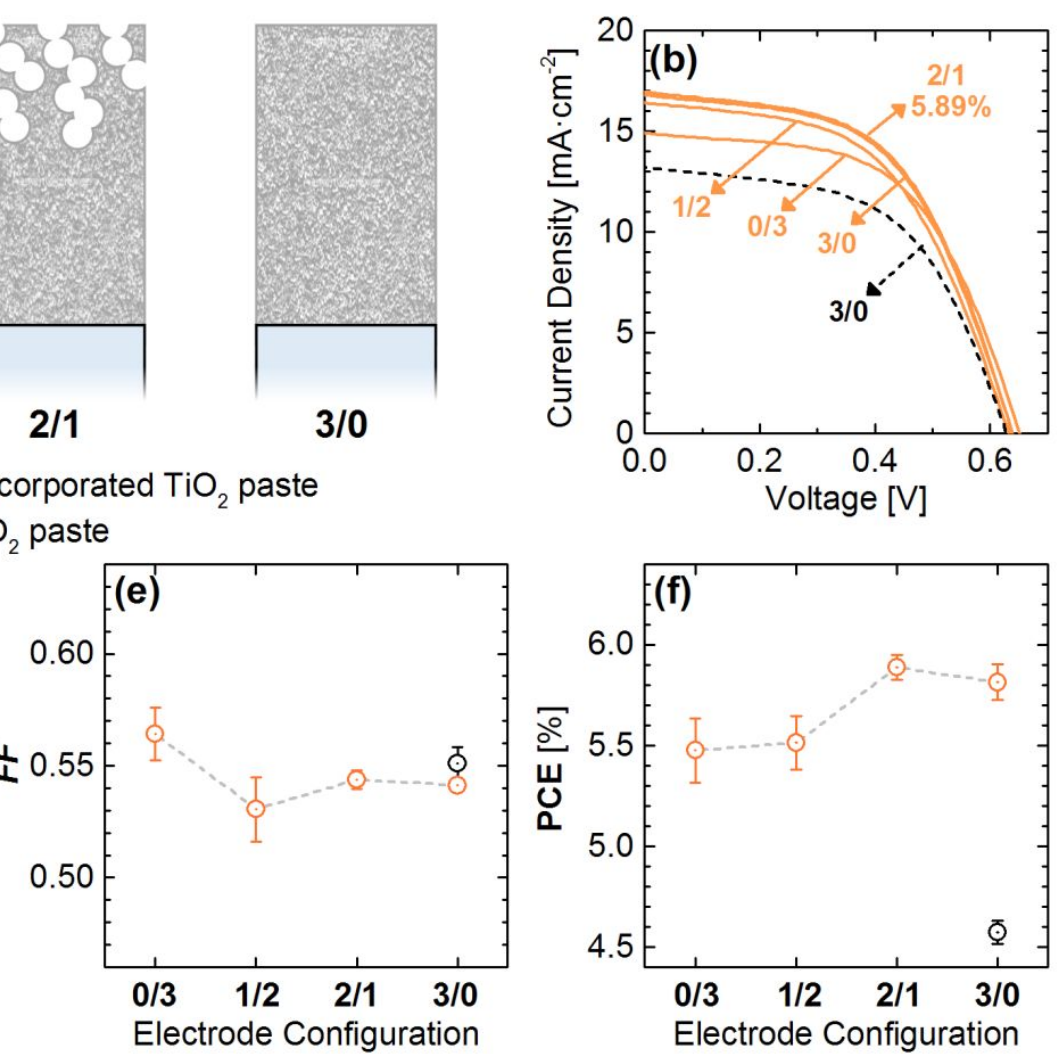

Figure S3. Performance of $6 \mathrm{CdS}-p \mathrm{HNR}(\mathrm{EP})-4 \mathrm{CdS}$ devices prepared with different $\mathrm{TiO}_{2}$ electrode configurations: (a) schematic illustration describing the electrode configurations (' $0 / 3$ ' configuration is the same that used to fabricate all the devices presented in main figures), (b) $J-V$ curves, and (c-f) the photovoltaic parameters. The electrophoretic deposition (EP) conditions applied here ( $10 \mathrm{~V}$ for $96 \mathrm{~h})$ are different from those applied to devices presented in main Fig. 3(d) ( $200 \mathrm{~V}$ for $20 \mathrm{~min}$; data presented in orange). When the EP conditions were same with those applied to devices presented in Fig. 3(d), devices from polystyrene-free electrodes resulted in far lower photovoltaic performance ('3/0' configuration; data presented in black). 
Table S1. Photovoltaic parameters of $6 \mathrm{CdS}-p \mathrm{HNR}(\mathrm{EP})-4 \mathrm{CdS}$ cosensitized solar cells fabricated using different electrode configurations presented in Fig. S3.

\begin{tabular}{c|c|c|c|c}
\hline $\begin{array}{c}\text { Electrode } \\
\text { Configuration }\end{array}$ & $\begin{array}{c}\boldsymbol{J}_{\boldsymbol{s c}} \\
{\left[\mathrm{mA} \cdot \mathrm{cm}^{-2}\right]}\end{array}$ & $\begin{array}{c}\boldsymbol{V}_{\text {oc }} \\
{[\mathrm{V}]}\end{array}$ & $\boldsymbol{F F}$ & $\begin{array}{c}\text { PCE } \\
{[\%]}\end{array}$ \\
\hline $0 / 3$ & $14.91 \pm 0.03$ & $0.65 \pm 0.01$ & $0.56 \pm 0.01$ & $5.48 \pm 0.16$ \\
\hline $1 / 2$ & $16.43 \pm 0.11$ & $0.63 \pm 0.00$ & $0.53 \pm 0.01$ & $5.51 \pm 0.13$ \\
\hline $2 / 1$ & $16.94 \pm 0.01$ & $0.64 \pm 0.00$ & $0.54 \pm 0.00$ & $5.89 \pm 0.06$ \\
\hline $3 / 0$ & $16.80 \pm 0.15$ & $0.64 \pm 0.01$ & $0.54 \pm 0.00$ & $5.81 \pm 0.09$ \\
\hline $3 / 0$ (black) & $13.21 \pm 0.07$ & $0.63 \pm 0.00$ & $0.55 \pm 0.01$ & $4.57 \pm 0.06$ \\
\hline
\end{tabular}

